\title{
Design, Assembly and Startup of a Single-Phase Multi-Modular Matrix Converter for Grid Interconnection
}

\author{
M. Rivera, G. Castro and P. Wheeler
}

\begin{abstract}
Power converters play a fundamental role in grid interconnection systems. In this article we present the design, assembly and start-up of a multimodular converter. The different stages and design components of the system are detailed. The main idea of this paper is to provide the reader with a guide on how to proceed with the design and assembly of this type of converters.
\end{abstract}

Index Terms-convertidores matriciales, convertidores de potencia, interconexión de redes.

\section{INTRODUCCIÓN}

Los convertidores de potencia juegan un importante rol para el manejo de la energía eléctrica, proporcionando la interconexión entre los sistemas de generación y distribución. Los convertidores de potencia usados comúnmente en la actualidad incluyen elementos almacenadores de energía los cuales agregan peso, tamaño y posibilidad de fallas. Los convertidores matriciales no tienen almacenadores de energía y ellos son una alternativa flexible y eficiente para el manejo de la energía [1].

Estos convertidores han sido considerados en aplicaciones específicas tales como militares, aeroespaciales, sistemas de generación eólica entre otros [2]-[5]. Sin embargo, ellos no han sido profundamente estudiados en aplicaciones para la interconexión de redes de sistemas de generación y/o cargas.

Está previsto que sus características: 1) circuito de potencia compacto y simple sin elementos almacenadores de energía; 2) capacidad de flujo bi-direccional; 3) generación de voltaje con amplitud y frecuencias arbitrarias; 4) generación de corrientes sinusoidales; 5) posibilidad de operación con desplazamiento unitario del factor de potencia, permita sistemas más robustos, con tamaños más pequeños y mejor desempeño que las configuraciones convencionales.

Existen distintas topologías de convertidores matriciales, una de ellas es la multinivel, la cual permite una distribución de potencia entre sus semiconductores con lo cual es posible controlar potencias mayores en base a semiconductores de menor potencia. Este concepto es posible utilizarlo en aplicaciones, tales como molinos de viento de gran escala que posean dichos sistemas en donde la confiabilidad y la tolerancia a fallas son de alto interés dado que se depende del clima para crear los espacios para la mantención y reparación [6].

M. Rivera and G. Castro are with the Faculty of Engineering, Universidad de Talca, Curico, Chile. (e-mail: marcoriv@utalca.cl, gcastro27.gc@gmail.com

P. Wheeler is with the Faculty of Engineering, The University of Nottingham, Nottingham, UK. (e-mail: Pat.Wheeler@nottingham.ac.uk
En este paper se desarrolla la idea de que es posible realizar la interconexión de diferentes tipos de sistemas de generación y/o cargas a través de una arquitectura multi-modular de conversión de energía, basado en la conexión en cascada de convertidores matriciales monofásicos con flujo de potencia bidireccional, de peso y tamaño reducido. Para permitir el flujo de potencia bidireccional entre diferentes sistemas de generación o cargas, es necesario que el convertidor que los interconecta tenga capacidad regenerativa. Un convertidor en cascada será bidireccional si cada celda es también bidireccional. Esto ya fue verificado en investigaciones previas usando puentes $\mathrm{H}$ o rectificadores PWM trifásicos en la entrada de cada celda. Ya que el convertidor matricial es intrínsecamente regenerativo, cada celda es automáticamente bidireccional por lo tanto no hay que preocuparse de este aspecto, el cual introduce una gran simplificación en el control. Además los convertidores matriciales son livianos y pequeños porque ellos no tienen elementos almacenadores de energía así el control del enlace CC no es usado y el riesgo de fallas es reducido.

\section{El Convertidor Matricial MultiModular MONOFÁSICO}

El convertidor matricial multi-modular monofásico indicado en la Figura 1, es la configuración propuesta en este trabajo. Esta topología consta de seis convertidores matriciales monofásicos (SPMC) conectados entre sí mediante transformadores de aislación galvánica de media frecuencia. Dicho transformador es el encargado de aislar la fuente de entrada con la carga. Este tipo de topologías se caracteriza por ser un convertidor CA/CA directo, que no posee elemento almacenador de energía lo que lo hace más pequeño que lo normal, y que gracias a que los transformadores usados son de frecuencia media, el tamaño de los mismos es menor. Este convertidor es capaz de entregar un voltaje de salida variable en amplitud y frecuencia y generar una corriente de entrada que está en fase con el voltaje de la red, mejorando así el factor de potencia del sistema [7], [8]. El elemento clave del convertidor matricial es el switch bidireccional completamente controlado el que puede operar en los cuatro cuadrantes, permitiendo el flujo de corriente y bloqueando el voltaje en ambas direcciones. Este switch permite operar a una alta frecuencia de conmutación. Debido a que este tipo de switch en la actualidad no existe como tal, éste se debe crear en base a un arreglo en antiparalelo de semiconductores. 


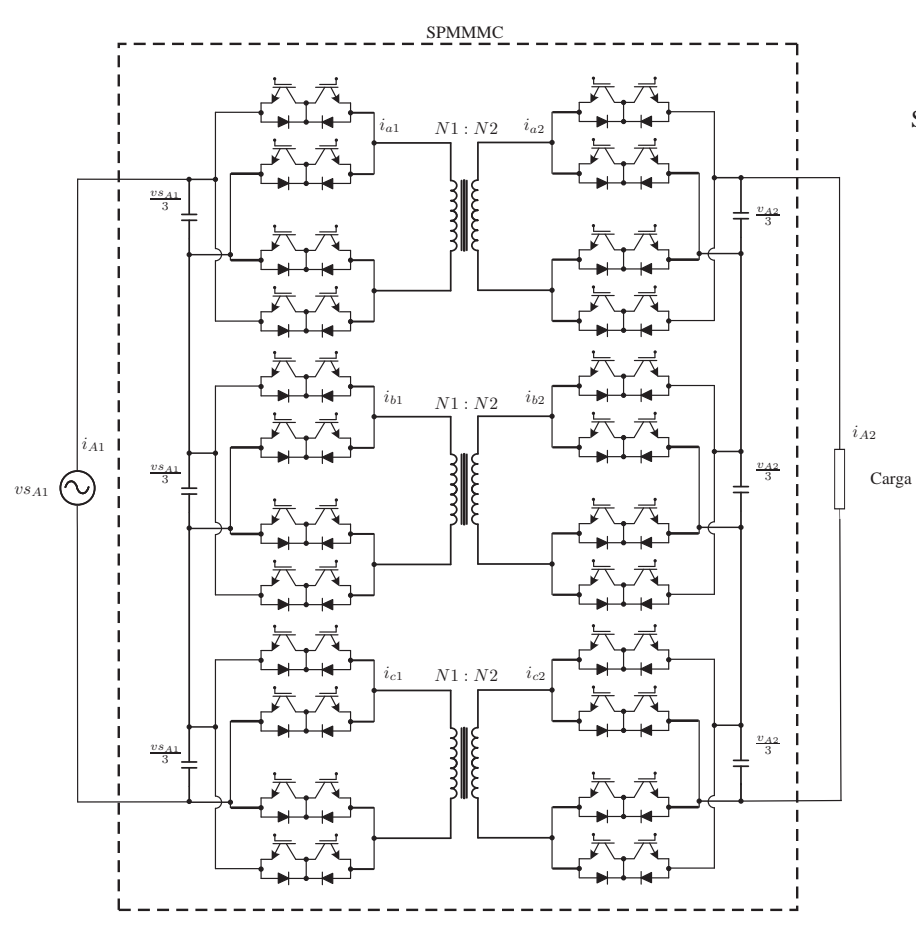

Fig. 1. Circuito propuesto para el convertidor.

El más usado en convertidores matriciales es el arreglo usando IGBT's y diodos para crear dicho circuito [9].

\section{Diseño, ARmado y Puesta en Marcha DeL CONVERTIDOR}

\section{A. Etapa de Potencia}

Los convertidores matriciales al no poseer elementos almacenadores de energía. El espacio que este tipo de estructura utiliza es menor en comparación a los convertidores que se encuentran en el mercado [10]. Es gracias a lo anterior que el convertidor matricial posee una densidad de potencia mayor que los otros convertidores, la cual llega a ser 5 veces mayor que otras topologías de convertidores [11], pero así como posee una densidad de potencia mayor la cantidad de semiconductores que necesita para operar también es mayor [9]. Esta característica lo hace ser una opción muy aplicada en el área aeroespacial.

En esta sección se muestra el funcionamiento y la estructura interna de las tarjetas de disparo y los switches utilizados.

1) Tarjeta de disparo: esta tarjeta es la que realiza la comunicación entre las señales de disparos que vienen desde la FPGA hacia los switches de potencia. Se debe tener en cuenta que esta tarjeta recibe una señal de voltaje en sus pines de entrada. Dichas señales de voltaje pasan a ser señales de luz en las salidas de la tarjeta. El esquema de bloques del funcionamiento interno de la tarjeta se aprecia en la Figura 2.

(a) Etapa conversor: esta etapa es la encargada de transformar la señal de voltaje de 3,3[V] (LVTTL, 0-3,3[V]) que proviene desde la salida FPGA a una señal de amplitud mayor de 5[V] (TTL, 0-5[V]). Esto se logra gracias a una resistencia PULL-UP, la cual realiza la función de elevador de voltaje.

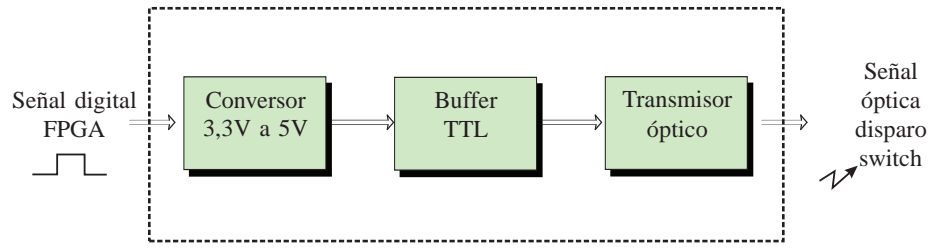

Fig. 2. Etapas dentro de la tarjeta de disparo.

(b) Etapa búffer TTL: esta etapa se incorpora al diseño para proteger a la FPGA de requerimientos excesivos de corriente. Esto se logra mediante el búffer, el cual es un arreglo de amplificadores operacionales conectados cada uno como seguidores, con lo que no admitirá corrientes altas desde las salidas de la FPGA, pero si puede entregar una mayor corriente en la salida del búffer (entrada de alta impedancia, salida de baja impedancia). Permitiendo funcionar únicamente con una señal de voltaje y en la salida entregando la corriente suficiente para el transmisor óptico.

(c) Transmisor óptico: esta etapa es la encargada de transformar la electricidad en luz. Esto se logra mediante la señal que proviene de la etapa anterior la cual pasa por un diodo LED de alta frecuencia. El mismo está encapsulado en un módulo adaptado para conectar fibra óptica, que es por donde se transmite la señal de disparo hacia los switches de potencia. En la Figura 3 se puede apreciar la tarjeta de disparo usada en el setup experimental.

2) Switch de potencia: (Fig. 4). El esquema de bloques del switch de potencia se puede apreciar en la Figura 5. Este switch tiene la característica de ser el elemento principal del convertidor, dado que entre sus terminales es por donde atraviesa toda la energía que el convertidor, además de que gracias a la interconexión de un conjunto de los mismos se logra la configuración multi-modular matricial.

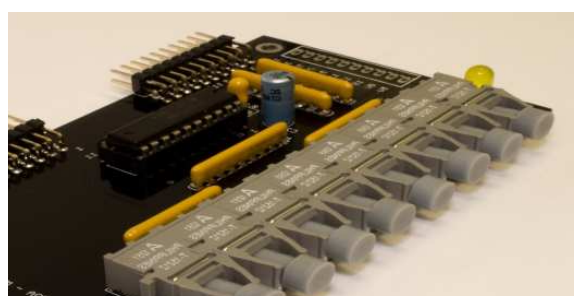

Fig. 3. Tarjetas de disparo usada en el setup experimental

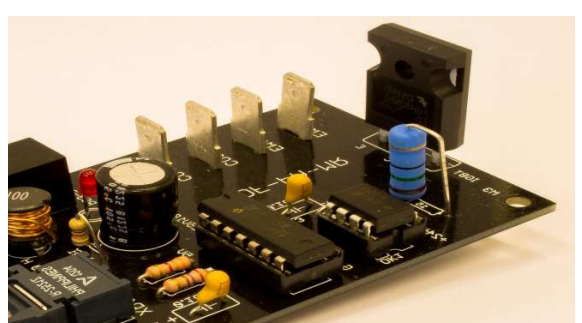

Fig. 4. Tarjeta del switch de potencia.

Esta tarjeta es la que realiza la conmutación de los IGBT's, el cual se encarga de permitir o negar el paso de la corriente 


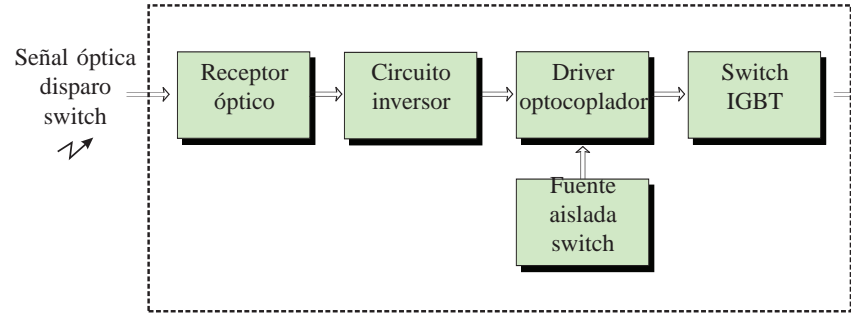

Fig. 5. Etapas dentro del switch de potencia

por sus terminales, con lo que se redirige la corriente dependiendo del estado que el controlador determinó.

(a) Etapa receptor óptico: esta etapa recibe la señal de luz que proviene desde la fibra óptica. Esto lo logra gracias a un módulo que tiene un transistor óptico el cual es el que transforma la señal de luz en una señal de voltaje, dado que el transistor dentro del módulo está en modo colector abierto se debe emplear una resistencia PULL-UP para que la señal de voltaje sea entregada de forma óptima.

(b) Etapa circuito inversor: en esta etapa la señal de voltaje que viene del receptor óptico se invierte, esto lo logra gracias a un circuito integrado que tiene compuertas NOT, entregando de esta forma una salida TTL negada a la de la entrada.

(c) Etapa driver optocoplador: esta etapa cumple la función de entregar al gate del IGBT la señal de disparo que controla la apertura o cierre del mismo. Este driver está compuesto por un optocoplador el cual es el encargado de aislar galvánicamente la señal de control con la señal que se le entrega al gate del IGBT.

Se debe tener en cuenta que toda la tarjeta del switch junto con el diodo emisor del optocoplador comparten una fuente de $5[\mathrm{~V}]$ a diferencia del transistor receptor el cual funciona con una fuente aislada, lo que permite al IGBT estar en un estado flotante (no posee una referencia impuesta). Esta etapa posee un diodo zener en la salida de la misma, el cual protege por sobre voltaje al gate del IGBT.

(d) Etapa fuente aislada: esta etapa es la que se encarga de entregar la alimentación al transistor receptor del optocoplador, con lo que se logra que el disparo hacia el gate del IGBT no posea ninguna referencia. Esta fuente está compuesta por un filtro de entrada y salida, e internamente es un convertidor CC/CC con aislación galvánica, además de poseer una protección contra corto circuitos.

(e) Etapa IGBT: esta etapa corresponde al switch que se encarga de realizar las conmutaciones de potencia, es un transistor del tipo IGBT el cual posee una alta impedancia de entrada, es de rápida conmutación, tiene una alta capacidad de corriente $(80[\mathrm{~A}], 600[\mathrm{~V}])$ y una baja saturación de voltaje colector-emisor.

\section{B. Etapa de Sensores y Acondicionador}

1) Mediciones y acondicionamiento de señales: para realizar el control del sistema se utilizaron tarjetas de sensado y acondicionado de señales. Dichas tarjetas de sensado miden tanto el voltaje como la corrientes del sistema y estas mediciones deben ser acondicionadas antes de llegar a las entradas de los controladores, ya sea la FPGA o DSP. Es para ello que se implementó el esquema que se aprecia en la Figura 6. En dicho esquema se logra diferenciar las etapas por las que se propaga cada señal, ya sea de corriente o de voltaje. En la primera etapa la amplitud de cada señal es medida por medio de un sensor ya sea de corriente o de voltaje, dicha señal es escalada en un rango de 4 a $20[\mathrm{~mA}]$, y esta señal de corriente llega a la segunda etapa (acondicionador de señales), esta etapa recibe la señal de corriente y la convierte en una señal de voltaje, con una amplitud máxima de 3,3[V], la siguiente etapa corresponde al controlador, el cual recibe la señal que internamente corresponde a una amplitud y es utilizada por el controlador para determinar mediante cálculos los vectores a aplicar en el convertidor.

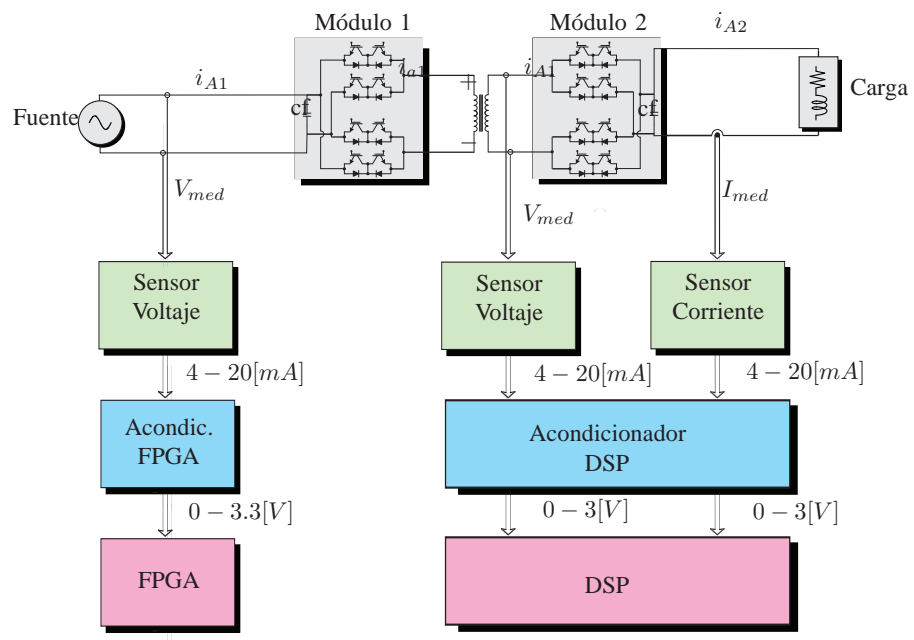

Fig. 6. Esquema de sensado y acondicionado de señales.

2) Sensor de voltaje: este sensor se encarga de tomar la medida de voltaje del convertidor y es sensado de forma diferencial. Dicho sensor internamente está dividido en distintas etapas, las cuales se aprecian en la Figura 7.

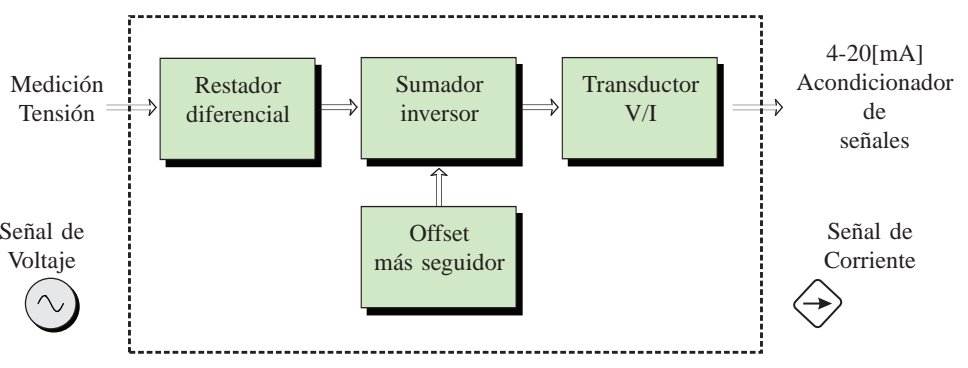

Fig. 7. Etapas dentro del sensor de voltaje para la DSP.

(a) Etapa restador diferencial: esta etapa se encarga de recibir la medida de voltaje directamente del convertidor. Esta etapa mide el voltaje diferencial entre dos puntos, siendo $600[\mathrm{Vpp}$ la medida máxima que se permite en la entrada. Dicha medida pasa por un circuito restador el cual utiliza amplificadores operacionales. Esta etapa entrega una señal en voltaje proporcional a la entrada. 
(b) Etapa sumador inversor: esta etapa corresponde a un amplificador operacional el cual recibe la señal del restador diferencial y la señal del offset, con lo cual la salida de esta etapa es una señal proporcional al voltaje de entrada, con la diferencia de que esta señal tiene un offset por lo que la señal se encuentra siempre en voltajes positivos.

(c) Etapa seguidor más offset: esta etapa entrega un offset a la señal de voltaje que se está manipulando. Para lograr este offset se utiliza un divisor de tensión cuyo voltaje de salida está conectado a un amplificador operacional conectado como seguidor, con lo cual se logra mantener constante este voltaje.

(d) Etapa transductor voltaje a corriente: esta etapa comprende dos transistores, un amplificador operacional y un arreglo de resistencias. Con todo esto se logra transformar la señal de voltaje proporcional de entrada con offset en una señal de corriente que varía entre 4 a $20[\mathrm{~mA}]$, siendo el offset de salida de $12[\mathrm{~mA}]$, la salida de esta etapa está conectada con el acondicionador de señales de la DSP.

3) Sensor de corriente: este sensor es el encargado de tomar la medida de corriente que pasa por la carga que está conectada en la salida del convertidor. Este sensor está dividido en distintas etapas, las cuales se aprecian en la Figura 8.

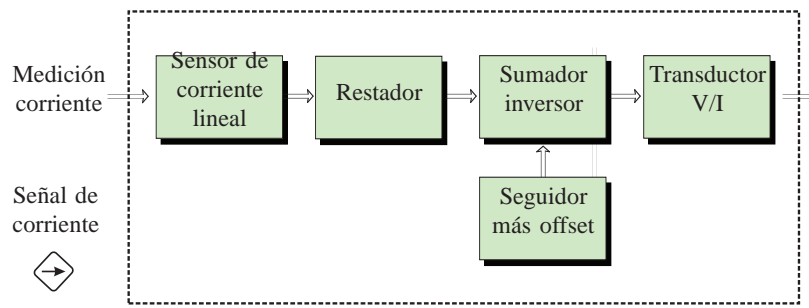
${ }_{\text {Acondic. }}^{4-20[\mathrm{~mA}]}$ Fig. 9. Etapas tarjeta acondicionadora de señales de la DSP. de señales

Señal de corriente $\rightarrow$

Fig. 8. Etapas dentro del sensor de corriente para la DSP.

(a) Etapa sensor de corriente lineal: esta etapa corresponde a un módulo de sensado de corriente, el cual tiene un sensor integrado que funciona mediante el efecto hall, lo que permite aislar el sistema de la etapa de sensado de señales.

Este sensor puede sensar como máximo 20[A] y entrega una señal de salida que varía entre 0 a $5[\mathrm{~V}]$. Esta señal de voltaje ya posee un offset, el mismo se encuentra en los 2,5[V].

(b) Etapa restador: esta etapa toma la señal de voltaje de la etapa anterior y la escala en voltaje para luego ser usada en las siguientes etapas. Esta señal, al pasar por esta etapa, elimina el offset de la etapa anterior.

(c) Etapa sumador inversor: esta etapa recibe dos señales, la que viene desde la etapa del restador y de la etapa del offset. Esta etapa se centra en tomar la señal proporcional a la entrada de corriente y a la misma agregar el offset deseado. Con esto, se obtiene en la salida una señal de voltaje proporcional a la corriente de entrada, en donde dicha señal tiene como punto nulo un voltaje de offset.

(d) Etapa seguidor más offset: esta etapa corresponde a un divisor de voltaje, con el cual se genera un voltaje constante gracias a un arreglo de resistencias y un amplificador operacional conectado como seguidor en la salida (lo que mantiene el voltaje de salida invariable). Este voltaje de salida se denomina offset y se usa en la etapa del sumador inversor.

(e) Etapa transductor voltaje a corriente: esta etapa corresponde a un conjunto de componentes que se encargan de transformar la señal de voltaje escalada que viene con un offset a una señal de corriente que varía entre 4 a $20[\mathrm{~mA}]$, y que tiene un offset de $12[\mathrm{~mA}]$. Esta etapa transmite su señal a la tarjeta acondicionadora de señales de la DSP.

4) Acondicionador de señales: esta tarjeta electrónica es la encargada de acondicionar las señales de los sensores tanto de voltaje como de corriente. Dicha tarjeta recibe señales en corriente y las escala a señales de voltajes óptimas para ser leidas por la tarjeta DSP. En la Figura 9 se aprecia el esquema de bloques de todas las etapas internas de la tarjeta acondicionadora de señales.

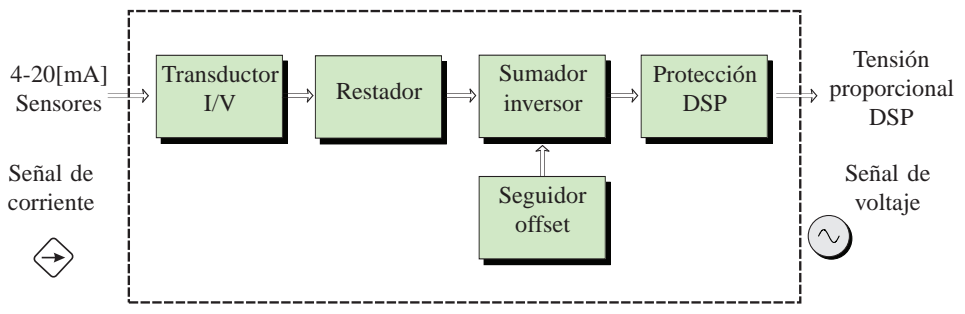

(a) Etapa transductor corriente voltaje: esta etapa es la encargada de transformar la señal de corriente que viene desde los sensores en una señal de voltaje. Esta etapa no es más que un arreglo de resistencias que al pasar la corriente por las mismas se produce un caída de tensión proporcional a las señales sensadas.

(b) Etapa restador inversor: esta etapa escala en voltaje la señal original además de eliminar el offset con el que dicha señal ya viene, con lo que entrega en su salida una señal en voltaje escalada y proporcional a la señal sensada originalmente.

(c) Etapa sumador inversor: esta etapa recibe tanto la señal que viene desde el restador como de la señal de la etapa del seguidor con offset, las cuales se suman y con ello se obtiene en la salida una señal en voltaje que varía entre $0-3[\mathrm{~V}]$ con un offset en los 1.5[V]. En esta etapa es en donde se termina de acondicionar las señales de sensado para ser leída en la DSP.

(d) Etapa seguidor más offset: en esta etapa se logra acondicionar un voltaje fijo para ser usado como offset por la etapa de sumador inversor. Esto se logra mediante un divisor de tensión hecho con un potenciómetro de precisión (lo cual permite variar el offset de la tarjeta a voluntad del usuario), en donde la salida de voltaje pasa por un amplificador operacional conectado como seguidor. Esto se realiza para mantener dicho voltaje de offset invariante.

(e) Etapa protección y salida: en esta etapa se agrega una protección física para proteger las entradas de la DSP, la señal pasa a través de un amplificador operacional conectado como seguidor usado para eliminar un poco la 
variación de voltaje y el ruido, y además pasa por un diodo zener el que limita el voltaje de salida del acondicionador de señales, en donde el voltaje de corte será de 3.3[V], esto se realiza para que al medir un sobre voltaje o sobre corriente que exceda a los rangos con los que se diseñaron los sensores la DSP no reciba en sus entradas analógicas voltajes mayores a los 3.3[V].

\section{Sensor de Voltaje para el Circuito de Protección Clamp}

Esta tarjeta es la encargada de sensar voltaje y entregar una señal proporcional a la FPGA. Dicho sensor está compuesto por un circuito integrado de sensado de voltaje, el cual es sensor lineal de voltaje LEM modelo LV25-P. Éste mismo tiene como salida una señal de corriente que varía entre 0$25[\mathrm{~mA}]$. El esquema por etapas del sensor se puede apreciar en la Figura 10.

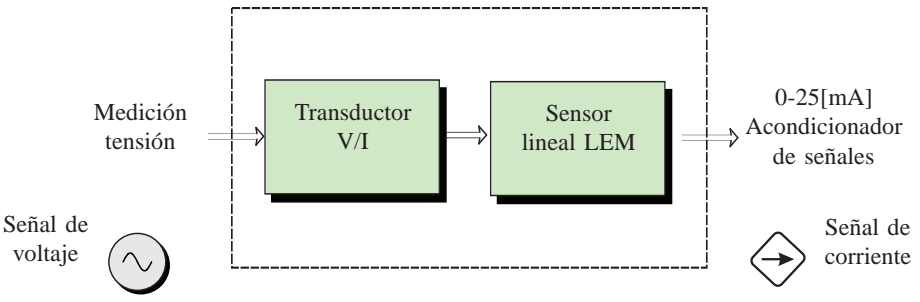

Fig. 10. Etapas dentro del sensor de voltaje para el circuito clamp.

(a) Etapa transductor voltaje corriente: esta etapa se encarga de escalar la señal del voltaje de entrada en una señal de corriente proporcional. Esto lo realiza mediante un arreglo de resistencias que para este caso tiene un valor 66 $[\mathrm{k} \Omega]$, al tener una diferencia de potencial en los terminales del arreglo de resistencias se logra una corriente que lo atraviesa. La corriente que se logra en la salida no debe superar los $10[\mathrm{~mA}]$, por lo que la máxima magnitud de voltaje que se puede medir es de 660[V].

(b) Etapa sensor lineal LEM: esta etapa corresponden únicamente a un sensor lineal encapsulado marca LEM módelo LV25-P, el cual es un transductor que utiliza del efecto hall para su funcionamiento. Este mismo requiere una corriente de entrada que no supere $\operatorname{los} 10[\mathrm{~mA}]$, con lo que entregará en su salida una corriente que varía entre $0-25[\mathrm{~mA}]$, teniendo un offset de salida en los 12,5[mA].

\section{Acondicionador de Señales para la FPGA}

Esta tarjeta posee dos canales, de los que cada uno tiene un propósito específico. Uno de ellos acondiciona la señal que proviene desde el sensor de voltaje de la FPGA y el otro canal se encarga de acondicionar la señal que proviene de la tarjeta del circuito clamp. Ambas señales son recibidas en un rango de corriente, el cual está entre 0-25[mA]. En la Figura 11 se aprecia el esquema de bloques de la tarjeta acondicionadora.

(a) Etapa transductor corriente a voltaje: esta etapa es común para ambos canales. En esta etapa la corriente que viene tanto desde el sensor de voltaje como del circuito de protección clamp pasan a través de un arreglo de resistencias para que se genere una caída de tensión entre

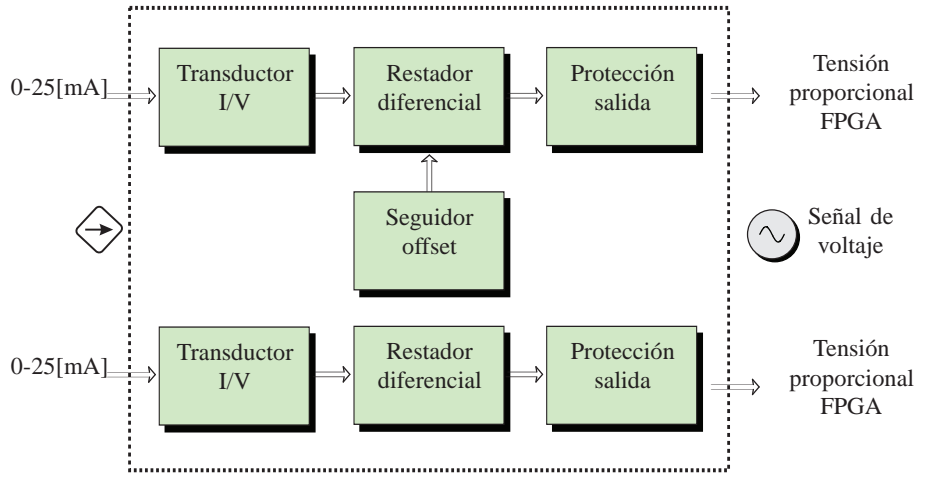

Fig. 11. Etapas tarjeta acondicionadora de señales de la FPGA.

sus terminales, con lo que se logra una señal de voltaje proporcional a la que entrega tanto el sensor de voltaje como el circuito clamp. En la salida de esta etapa se tiene una señal de voltaje que varía entre 0-5[V].

(b) Etapa restador diferencial: esta etapa también es común para ambos canales. Aquí se comparan voltajes con respecto a uno de referencia, para el caso del circuito clamp este voltaje es $0[\mathrm{~V}]$ dado que solo se reciben voltajes positivos. Para el caso del sensor de voltaje, la tensión con la que se compara es el que viene de la etapa de seguidor más offset, dado que la señal de voltaje varía entre valores negativos a positivos. La salida de esta etapa en ambos casos entrega un voltaje proporcional al sensado en las entradas, el cual varía entre 0-3,3[V].

(c) Etapa seguidor con offset: esta etapa es la encargada de generar el voltaje de offset que se requiere para el canal donde está conectado el sensor de voltaje. Dicho voltaje se logra con un divisor de tensión hecho de resistencias fijas y un amplificador operacional conectado como seguidor en la salida del divisor de tensión. Esta etapa entrega un voltaje constante e invariable en el tiempo.

(d) Etapa protección y salida: esta etapa es común para ambos canales. Es aquí donde se interconecta la salida de la etapa de restador diferencial con la FPGA. El voltaje que viene de la etapa anterior pasa un diodo zener, el cual se encargará de recortar la señal cuando ésta supera los 3,3[V]. La salida de esta etapa se conecta directamente con un módulo acondicionador de señales especial para la FPGA llamado PmodAD1, el cual es un módulo de dos entradas y que entrega un dato de 12 bits por cada entrada.

\section{E. Programación y comunicación de la DSP y FPGA}

Para la operación segura de este convertidor se considera una DSP y una FPGA. Ambas plataformas digitales se programaron por separado pero entre sí requieren mantener una comunicación en todo momento. Por límite de páginas en este artículo, no se detallarán todos los módulos de estas plataformas.

En particular, la DSP es la encargada de recibir las mediciones de los sensores de corriente y voltaje, así como también la interrupción proveniente de la FPGA para ejecutar el algoritmo de control. A su vez, la DSP envía a la FPGA el estado de conmutación a aplicar en el siguiente instante de 
muestreo. La FPGA, por su parte, es la encargada de generar la señal de interrupción hacia la DSP, así como también de leer el estado a aplicar al convertidor. Además, la FPGA es la encargada de asegurar el correcto funcionamiento del convertidor, ejecutando la conmutación segura de éste. El circuito clamp es controlado también por la FPGA, abriendo los dispositivos de conmutación en caso de existir algúna sobre tensión o cortocircuito en el convertidor.

\section{EVALUACIÓN EXPERIMENTAL}

En la Figura 12 se aprecian los resultados experimentales del convertidor, se obtuvieron con un voltaje en la entrada de 113 [Vpp] (80[Vrms]), con una carga resistiva de $20[\Omega]$ y un inductor de $10[\mathrm{mH}]$.
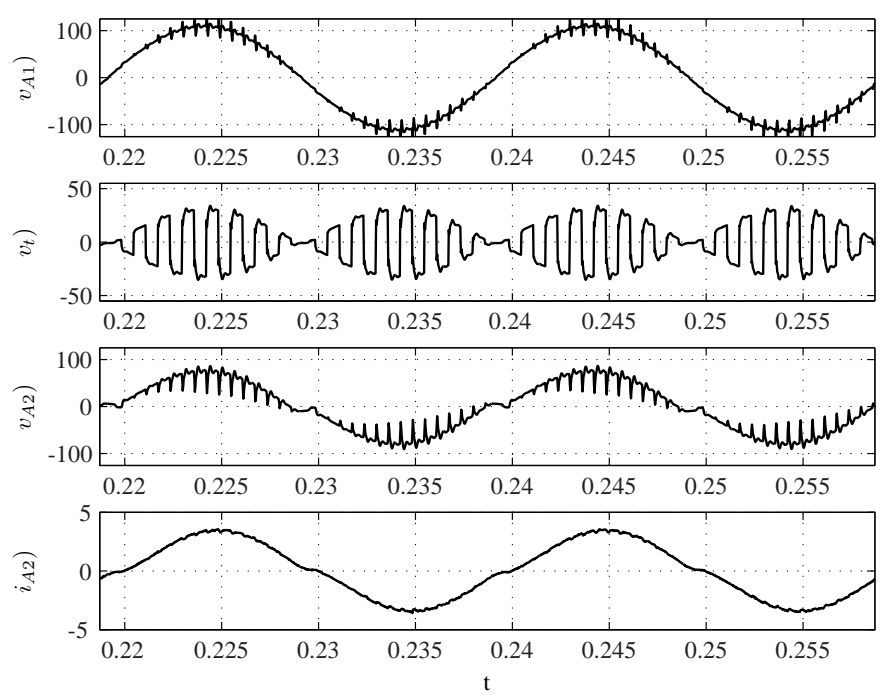

Fig. 12. Resultados experimentales del convertidor considerando tres celdas $(\mathrm{SPMC}+\mathrm{IT}+\mathrm{SPMC})$ en cascada, 113[Vpp] entrada, carga de $20[\Omega]$ y un inductor de $10[\mathrm{mH}]$.

En los resultados experimentales se logra ver que los peak's de voltaje generados por las inductancias parásitas no son menores, aunque la forma general del voltaje es la deseada, las pérdidas por conducción son muy altas, por lo mismo

\section{AGRADECIMIENTOS}

Los autores agradecen el apoyo financiero de FONDECYT Regular 1160690 y el Proyecto de Atracción de Capital Humano Avanzado: Modalidad Estadías Cortas 80150056. no se logra aprovechar la potencia como se desearía, ya que una buena parte de la misma se disipa o se pierde en los semiconductores, cables o transformadores.

\section{COnClusiones}

En este artículo se logró un análisis de los aspectos más importantes en la construcción de un convertidor multimodular monofásico, para el uso en la integración de redes. Se han descrito los diferenets circuitos utilizados para su puesta en marcha, y se han presentado los diferentes módulos que componen cada sistena. Con esto, la idea es dar al lector una visión general de los diferentes aspectos a considerar a la hora de diseñar un convertidor de potencia.

\section{REFERENCES}

[1] J. Rodriguez, M. Rivera, J. W. Kolar, and P. W. Wheeler, "A review of control and modulation methods for matrix converters," IEEE Transactions on Industrial Electronics, vol. 59, no. 1, pp. 58-70, Jan 2012.

[2] L. de Lillo, L. Empringham, P. Wheeler, J. Clare, and K. Bradley, "A $20 \mathrm{kw}$ matrix converter drive system for an electro-mechanical aircraft (ema) actuator," in 2005 European Conference on Power Electronics and Applications, Sept 2005, pp. 6 pp.-P.6.

[3] P. W. Wheeler, J. C. Clare, M. Apap, L. Empringham, L. D. Lilo, K. Bradley, C. Whitley, and G. Towers, "An electro-hydrostatic aircraft actuator using a matrix converter permanent magnet motor drive," in Second International Conference on Power Electronics, Machines and Drives (PEMD 2004), vol. 2, March 2004, pp. 464-468 Vol.2.

[4] C. Klumpner, P. Nielsen, I. Boldea, and F. Blaabjerg, "A new matrix converter motor $(\mathrm{mcm})$ for industry applications," IEEE Transactions on Industrial Electronics, vol. 49, no. 2, pp. 325-335, Apr 2002.

[5] P. W. Wheeler, J. C. Clare, M. Apap, D. Lampard, S. J. Pickering, K. J. Bradley, and L. Empringham, "An integrated 30kw matrix converter based induction motor drive," in 2005 IEEE 36th Power Electronics Specialists Conference, June 2005, pp. 2390-2395.

[6] D. Karwatzki, M. von Hofen, L. Baruschka, and A. Mertens, "Operation of modular multilevel matrix converters with failed branches," in IECON 2014 - 40th Annual Conference of the IEEE Industrial Electronics Society, Oct 2014, pp. 1650-1656.

[7] A. Alesina and M. Venturini, "Intrinsic amplitude limits and optimum design of 9-switches direct pwm ac-ac converters," in Power Electronics Specialists Conference, 1988. PESC '88 Record., 19th Annual IEEE, April 1988, pp. $1284-1291$ vol.2.

[8] A. Alesina and M. G. B. Venturini, "Analysis and design of optimumamplitude nine-switch direct ac-ac converters," IEEE Transactions on Power Electronics, vol. 4, no. 1, pp. 101-112, Jan 1989.

[9] P. W. Wheeler, J. Rodriguez, J. C. Clare, L. Empringham, and A. Weinstein, "Matrix converters: a technology review," IEEE Transactions on Industrial Electronics, vol. 49, no. 2, pp. 276-288, Apr 2002.

[10] A. Alesina and M. Venturini, "Solid-state power conversion: A fourier analysis approach to generalized transformer synthesis," IEEE Transactions on Circuits and Systems, vol. 28, no. 4, pp. 319-330, Apr 1981.

[11] G. Roy and G. E. April, "Direct frequency changer operation under a new scalar control algorithm," IEEE Transactions on Power Electronics, vol. 6, no. 1, pp. 100-107, Jan 1991. 